\title{
El paisaje como
}

\section{RECURSO TURÍSTICO en las áreas naturales protegidas: caso reserva de la Biosfera Janos, Chihuahua, México}

Rosa Herminia Suárez Chaparro Universidad Autónoma de Ciudad

Juárez
Recibido: 30 de abril de 2014

Aceptado: 10 de septiembre de 2014

\section{RESUMEN}

aisaje, áreas naturales y turismo son conceptos interrelacionados. El paisaje determina la visitación de los turistas en áreas naturales y puede llegar a ser uno de los recursos más valiosos para la consolidación y diseño de la oferta turística, sin dejar de señalar que es un recurso delicado puesto que, una vez que se deteriora o se altera, su recuperación es muy compleja y en muchos de los casos imposible. Asimismo, el paisaje es más difícil de administrar comparado con otros servicios que demandan los turistas en áreas naturales.

En este estudio se analiza la valoración del paisaje como recurso integrador de la oferta turística, el cual se ha basado en encuestas y entrevistas aplicadas a visitantes y residentes en el Área Natural
Protegida Reserva de la Biosfera Janos, Chihuahua, México, con el objetivo de orientar una gestión turística sostenible.

Los resultados que se presentan corresponden al trabajo de campo llevado a cabo durante el periodo octubre-diciembre de 2013. Es de destacar la participación de visitantes y residentes en la valoración de atributos del paisaje a través de 10 fotografías de los lugares más representativos del área de estudio.

Palabras clave: paisaje, áreas naturales, turismo, Janos, México.

\section{Abstract}

T andscape, natural areas and tourism are interrelated concepts. The landscape determines the tourists visiting in natural areas and it can be one of the most valuables resources to the consolidation and design of 
the tourist offer and still pointing that the landscape is one delicate resource because when is deteriorated or altered need a very complex restoration, even in many cases is impossible. Furthermore the landscape is more difficult to manage than other touristic services demanded by tourists in natural areas.

This study analyzed a landscape valorization like an integrator resource in the tourist offer, which is based on surveys with in tourists and residents' interviews in the Janos National Nature Reserve (JNR), located in Chihuahua, in the north of Mexico. The aim of this study is to guide the planning of territorial use in this area to obtain a Sustainable Tourism Management (STM).

The results presented correspond to fieldwork performed during the period October to December 2013, emphasizing the participation of visitors, tourists and residents in evaluating the landscape through 10 photographs selecting the most spectacular, interesting and pleasant landscape.

Keywords: landscape, natural areas, tourism, Janos, México.

\section{INTRODUCCIÓN}

U n buen número de espacios o áreas naturales compiten por ofrecer al turista una diversidad de actividades y servicios, donde se tiende a buscar la diferenciación de la oferta turística por medio de la especialización o la puesta en valor de los recursos atractivos turísticos relacionados fundamentalmente con la cultura y la naturaleza. En los últimos años se ha dado un creciente desarrollo de la actividad turística en las áreas naturales protegidas. Los resultados en investigaciones mencionan que el $15 \%$ y el $30 \%$ de los ingresos totales del turismo a nivel mundial provienen del turismo de naturaleza. Asimismo, los datos estadísticos arrojan que el $40 \%$ de los norteamericanos programaron en el año 2000 viajes que tenían relación con la naturaleza y, para aproximadamente 20 millones de europeos, su principal motivación turística son los viajes en contacto con la naturaleza (Budowski, 2000 en Del Risco \& Salinas, 2003). El informe de la Comisión Europea (2002), Using Natural and Cultural Heritage to Develop Sustainable Tourism muestra que para un $50 \%$ de los europeos encuestados el paisaje es el criterio más relevante a la hora de elegir el destino turístico (Pintó, 2005), por lo que estos espacios naturales han pasado de ser un simple atractivo de la actividad turística para convertirse en destinos turísticos más solicitados por quienes buscan experiencias y actividades en un entorno natural con alto valor ecológico y paisajístico.

El objetivo de este trabajo de investigación es incorporar el valor del paisaje como recurso integrador de la oferta turística, que sirva de base para una propuesta de gestión del turismo en el Área Natural Protegida (ANP) Reserva de la Biosfera Janos (RBJ).

\section{ANTECEDENTES}

$\mathrm{E}$ 1 paisaje ha sido abordado desde diferentes perspectivas, se han cruzado miradas desde el punto de vista de la historia del arte, la estética, la literatura, el urbanismo, la ecología, la arqueología, la geografía, y muchas de sus definiciones están condicionadas por una instrumentalización que deriva de demandas sociales, económicas o políticas, desde los poderes públicos (Maderuelo, 2005).

El Convenio Europeo del Paisaje, aprobado por el Consejo de Europa en Florencia el año 2000, es el documento de referencia en cuanto a las políticas de paisaje en el marco de la Unión Europea, el cual considera al paisaje como un com- ponente esencial del entorno de la gente, una expresión de la diversidad de su patrimonio cultural y natural compartido, y un fundamento de su identidad (art. 5). Asimismo, reconoce la estrecha relación existente entre paisaje y turismo, el papel central del paisaje en el desarrollo del turismo sostenible, y lo considera un elemento esencial para el logro de un equilibrio entre la preservación del patrimonio natural y cultural como reflejo de la identidad y diversidad europeas, además de un recurso económico capaz de generar trabajo en un contexto de incremento del turismo sostenible (Pintó, 2005).

\section{El paisaje Y SU RELACIÓN CON EL TURISMO

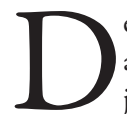 entro de la clasificación de los atractivos turísticos, el paisa- je se convierte en uno de los} recursos valiosos para la consolidación de la oferta turística, acotando que es el recurso más delicado, comparado con la hostelería y las instalaciones o facilidades para el entretenimiento, recreación y diversión. El paisaje es más difícil de administrar y además su recuperación, una vez que este ha sido alterado o deteriorado, es muy costosa y en la gran mayoría de los casos imposible (Nogué, 1989).

El turismo recurre al paisaje como recurso, determina los lugares de interés que habrán de visitarse, pero no solo los turistas o visitantes evalúan la calidad del paisaje o lo atractivo del paisaje, sino la comunidad anfitriona, que modifica, transforma o preserva el paisaje (Bertocello, 2009 en Souto, 2011), integrando con ello valor social y aportando criterios para su valoración como elemento crucial de los recursos turísticos. Este valor que se le asigna por razones ambientales, sociales, culturales o visuales darán como 
resultado una mejor ordenación del territorio y, sobre todo, un entendimiento de los vínculos que existen entre los paisajes y las personas (Iglesias, 2010), en función de su belleza escénica, visual y cultural.

La interacción entre turismo y paisaje es evidente. La actividad turística está basada en la atracción de los paisajes y estos a su vez se convierten en un bien de consumo gracias al turismo (Gros, 2002). En entrevista, el Director del Observatorio del Paisaje de Catalunya, Joan Nogué I Font, declara que el "paisaje no es sólo un valor añadido, sino el principal valor de los lugares que viven del turismo", por lo que es muy clara su relación, sobre todo en la actualidad, donde el fenómeno de la globalización incide en la decisión de los turistas de donde pasar sus vacaciones, a un costo cada vez más competitivo. Entonces, lo único que queda al destino turístico es mostrar su autenticidad y en buena medida esto se concreta a través del paisaje (Ferrer, 2010).

Se debe tener presente que el paisaje ha de ser valorado y cuidado para reforzar el sentimiento de pertenencia de la comunidad que reside en él, ya que el turismo actúa como un arma de doble filo: por un lado puede llegar a apreciar y valorar el paisaje y por otro sólo convertirlo en objeto de consumo, sin ninguna consideración, transformando el lugar en un espacio monótono y gravemente modificado (Gros, 2002).

\section{RELACIÓN TURISMO Y ÁREAS NATURALES}

T as áreas naturales protegidas tienen como propósitos mantener 1 los ecosistemas naturales operativos, actuar como refugios para las especies, preservar los procesos ecológicos y cumplen además como indicadores que permiten entender las interacciones humanas con el mundo natural, es decir, constituyen la última esperanza con la que se cuenta para impedir la extinción de muchas especies amenazadas o endémicas (Dudley, 2008). Estas brindan beneficios no solamente ecológicos, sino también económicos, sociales e institucionales (López, 2012). Son fundamentales para el desarrollo sostenible de comunidades, especialmente indígenas o rurales, que dependen de los bienes y servicios ambientales que brindan para su supervivencia; son importantes para la investigación, la educación y contribuyen a las economías locales y regionales a través de actividades económicas como el turismo. Esta importancia es reconocida por el Convenio sobre la Diversidad Biológica $(\mathrm{CBD})$ y además busca proteger la belleza escénica de sus paisajes naturales o culturales, la diversidad biológica, el desarrollo de la investigación y contribuir con los objetivos de la sostenibilidad mediante la educación ambiental en las propias comunidades (UICN, 2009).

A finales de los años noventa y principios de este siglo, las ANP se han convertido en lugares preferidos para disfrutar del tiempo libre y en destinos turísticos, sin embargo, no todas las actividades que se realizan en ellas tienen el propósito de proteger y conservar el espacio natural. Es por ello que se promueve mantener una estrecha relación con algunas iniciativas de impulso del turismo de naturaleza, como la Carta Europea del Turismo Sostenible, la cual se ha posicionado como el instrumento más pujante y, seguramente, el más adecuado para la protección y conservación de las áreas naturales (Muñoz, 2008). Con esto habrán de considerarse las premisas que permitan la convivencia del uso racional y la conservación de los recuros naturales y culturales existentes mediante activida- des de turismo de bajo impacto, como lo es el Ecoturismo (Millán, 2001).

\section{TURISMO Y ÁrEAS Protegidas EN MÉXICO}

$\mathrm{M}$

éxico se destaca como uno de los diez destinos más visitados del mundo (cerca de 20 millones de turistas al año), lo que hace del turismo un importante generador de empleo directo e indirecto. Unos siete millones de esos turistas visitan los parques nacionales y otras áreas protegidas, en espacios fundamentales de la oferta turística nacional (Guerrero, 2011).

La actividad turística en las Áreas Naturales Protegidas (ANP) está regulada por la Comisión Nacional de Áreas Protegidas (ConAnp), que desde el 2000 ha impulsado una estrategia orientada a la sostenibilidad conocida como la "Estrategia nacional para un desarrollo sustentable del turismo y la recreación en las Áreas Naturales Protegidas de México", cuyo objetivo es contribuir a la conservación del patrimonio natural y cultural de las ANP impulsando actividades de turismo de bajo impacto a través de la aplicación de instrumentos de planeación, regulación y vigilancia (Conanp, 2000). Esta estrategia se lleva a cabo conjuntamente con la Secretaría de Turismo (Sectur).

\section{ÁREA DE ESTUDIO}

T a Reserva de la Biosfera Janos se localiza en la parte noroeste del estado de Chihuahua, al sur de la frontera con Estados Unidos y al este del estado de Sonora, en las Provincias de Sierras y Llanuras del Norte y Sierra Madre Occidental y en las Subprovincias de Llanuras y Médanos del Norte y Sierras y Cañadas del Norte, (Longitud $108^{\circ}$ 
56' 49.1712"; Latitud 31 11' 7.6344"). Se encuentra en el municipio de Janos y abarca casi toda la superficie del mismo (Conanp, 2013).

La superficie total de la reserva es de 526 mil 482-42-66.80 hectáreas.(CoNANP, 2013). De conformidad con lo previsto en el artículo 58 de la Ley General del Equilibrio Ecológico y la Protección al Ambiente (LGEEPA), el 8 de diciembre del 2009 se declara área natural protegida, con el carácter de reserva de la Biosfera (Conanp, 2013).

La Reserva de la Biosfera Janos es la primera ANP dedicada a la protección de pastizales (Fig. 2), los cuales representan el $42.4 \%$ de la superficie de la reserva, se distribuyen en la parte central de la misma, especialmente en terrenos planos con lomeríos, y aun cuando se encuentran sobrepastoreados, mantienen la mayoría de las especies originales, como son los perritos llaneros, que se encuentran bajo la categoría Amenazada (A) (CoNANP, 2013).

las amenazas a los perros llaneros en el área son el envenenamiento y la conversión de pastizal natural a cultivos (Pacheco, Ceballos, \& List, 2000).

El municipio de Janos se compone de 102 localidades, caseríos o centros poblacionales, todos ellos rurales $(\mathrm{Mu}-$ nicipio de Janos, Chih., 2010). Sus pobladores presentan un mosaico cultural (Manzano \& List, 2006), su población total es de 10953 habitantes, $47.7 \%$ corresponde a mujeres y el $52.3 \%$ corresponde a hombres (INEGI, 2011). La mayor parte de la población se dedica al sector primario.

El turismo también es una actividad que se desarrolla en la región, sin embar- go su impacto económico no ha tenido repercusiones favorables en la comunidad. El estudio realizado por Cuevas (2006) pone de manifiesto el gran vacío de productos turísticos que pudieran generar el interés por visitar la zona. Asimismo, se señala en dicho estudio que esto se debe a la falta de cooperación o colaboración de los municipios aldeanos a Janos, así como a la falta de apoyo a nivel estatal y federal para los pequeños propietarios que ofrecen servicios de hospedaje y alimentación (Cuevas, 2006). Pero el Plan Municipal de Desarrollo 2010-2013, contempla líneas de acción y estrategias para apoyar el desarrollo turístico sustentable desarrollando un corredor turístico del desierto a la sierra en la región noroeste del estado, a través de la promoción de convenios de coordinación y asignación de recursos (Municipio de Janos, Chih., 2010).

\section{Metodología}

$\mathrm{L}$ a metodología propone un estudio interpretativo-cualitativo utilizando fotografías de entornos paisajísticos del área y aplicar cuestionarios a turistas y/o visitantes, residentes, complementando la evaluación con entrevistas a funcionarios y residentes.

Se programó una visita a la RBJ previa autorización del Director del ANP. El trabajo se dividió en tres etapas: 1) Se realizó un recorrido de observación y reconocimiento de la zona aprovechando para tomar fotografías de los paisajes más representativos del área, específicamente de las áreas de uso público y turístico: Ejido Casa de Janos; 2) Para obtener información se aplicaron cuestionarios a visitantes y residentes de la RBJ, así como la realización de entrevistas a residentes; 3) Se organizó un taller participativo, cuyo objetivo era conocer la opinión de residentes y encargados de la protección y conservación del área para implementar un modelo de gestión de turismo sostenible.

Los cuestionarios se aplicaron in situ y de forma electrónica, los primeros fueron aplicados a visitantes y residentes durante los meses de octubre a diciembre del 2013 y los segundos fueron enviados - muestra aleatoria de personas - por correo electrónico y redes sociales (Facebook y Twitter), obteniendo un total 96 encuestas. Los cuestionarios se estructuraron en tres partes: 1) para definir el perfil del turista/visitante (por qué razón visitaba el lugar, quién lo acompaña en sus viajes, nacionalidad, lugar de residencia, escolaridad); 2) para determinar la preferencia de instalaciones o servicios turísticos en las ANP por parte de los visitantes/turistas (seguridad, servicios, para disfrutar de la estancia, especializadas); 3) para evaluar el paisaje por medio de fotografías, que fueron tomadas en una visita previa; en la selección de fotografías de la encuesta participaron residentes, visitantes, funcionarios, académicos y expertos en análisis del paisaje.

\section{RESULTADOS}

1 cuestionario fue respondido por
96 personas ( $51 \%$ son menores
de 30 años, el $49 \%$ de 31 a 60 años). El 66\% son mujeres y el $34 \%$ son hombres. En cuanto a su lugar de residencia, el $67 \%$ son residentes del estado de Chihuahua y el porcentaje restante son de otras entidades de la República Mexicana y extranjeros. Por último, el $68 \%$ tiene nivel de estudios de licenciatura y el 16\% de posgrado (ver tabla 1 ). 
Tabla 1. Perfil del turista/visitante

\begin{tabular}{|l|l|l|}
\hline Edad & Frecuencia & Porcentaje \\
\hline Menos de 30 & 49 & $51 \%$ \\
\hline De 30 a 60 & 47 & $49 \%$ \\
\hline Más de 60 & 0 & \\
\hline \multicolumn{2}{|c|}{ Género } \\
\hline Masculino & 33 & $34 \%$ \\
\hline Femenino & 62 & $66 \%$ \\
\hline & Lugar de residencia \\
\hline Chihuahua & 88 & $92 \%$ \\
\hline Otros estados & 2 & $2 \%$ \\
\hline Extranjero & 6 & $6 \%$ \\
\hline & Escolaridad & \\
\hline Primaria & 0 & $2 \%$ \\
\hline Secundaria & 2 & $12 \%$ \\
\hline Prepa/bachiller & 12 & $68 \%$ \\
\hline Licenciatura & 65 & $16 \%$ \\
\hline Posgrado & 15 & $2 \%$ \\
\hline Otra & 2 & \\
\hline
\end{tabular}

Fig. 1 ¿Por cuáles de los siguientes motivos

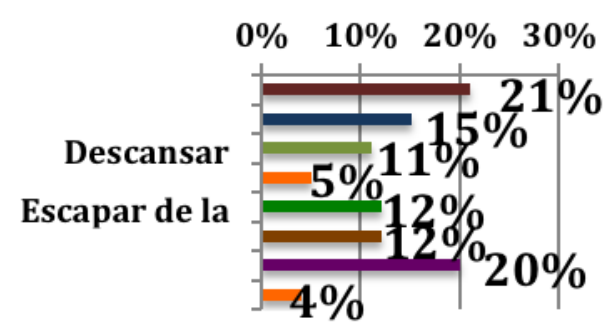

Fig. 2 ¿Quién lo acompañaría en su visita visitaría el ANP? (puede elegir más de una opción).

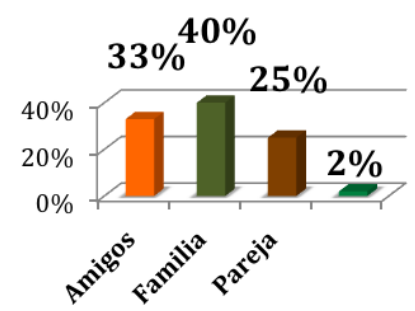

Fuente: Elaboración propia

En la pregunta ¿Cuál sería el motivo para visitar el ANP? (fig. 1) las opciones mayormente señaladas son: "por su belleza natural", con un $21 \%$; "estar en contacto con la naturaleza", el 20\%; "disfrutar el tiempo libre con amigos y familiares”, 15\%. En la pregunta ¿Quién lo acompañaría en su viaje?, el 57\% de los encuestados respondió que su pareja, el $47 \%$ amigos y el $37 \%$ su familia (fig. 2).

Las instalaciones que mayormente eligieron para disfrutar son: casetas de información y señalización, senderos para caminatas, áreas para acampar y preparar alimentos, hospedaje; mientras que los servicios que más se busca en esta área son: visitas guiadas, venta de artesanías, renta y venta de equipo ( fig. 3). 
Fig. $3 \gtreqless$ Cuáles de las siguientes instalaciones y/o servicios turísticos considera necesarios para disfrutar su visita en el ANP?

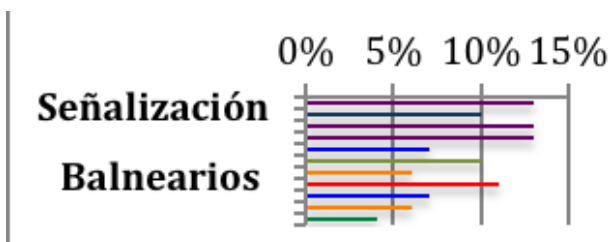

Fuente: Elaboración propia.

Una pregunta importante para este estudio corresponde a la aceptación de los visitantes de cubrir costos por la visita y el uso del área. El resultado es satisfactorio ya que en su mayoría están dispuestos a pagar cantidades entre 20 y 100 pesos mexicanos, incluso el $58 \%$ pagaría 50 pesos mexicanos por visita y por persona, cantidad que en opinión de los encuestados debe ser destinada a la conservación del paisaje y los recursos naturales.

\section{Evaluación del PAISAJE}

7 sta parte del cuestionario contiene: 10 fotografías de -1 paisajes representativos de la ANP ordenados de la "A" a la "J" y una lista de adjetivos jerarquizados en la idea de Craik (1975) y adaptados por Muñoz-Pedreros et al. (1993) a las características culturales y especialmente conceptuales de la población (Muñoz-Pedreros, 2004). Los adjetivos que permiten evaluar el paisaje observado en las fotografías fueron: Espectacular; Estimulante; Interesante; Agradable; Sencillo; Conservado; Sin Interés; Desagradable (agrupados según la escala universal de Fines).

Las imágenes fueron seleccionadas de más de 650 fotografías tomadas en el ANP. Esta selección se hizo con base en la opinión del personal de la ConANP, estudiantes y residentes del Ejido Casa de Janos, académicos y expertos en análisis del paisaje, así, los encuestados calificarían directamente cada fotografía. En la tabla 2 se presentan los resultados de la valoración, donde destacan las fotografías E, F y H con el adjetivo de Espectacular; Estimulante corresponde a la imagen D y los paisajes valorados como Agradables son las fotografías I, A y J. Cabe señalar que en esta valoración de los atributos mayormente se seleccionaron aquellas fotografías en las que el valor más significativo tiene que ver con los cuerpos de agua. 
Tabla 2. Resultados de la evaluación del paisaje de la Reserva de la Biosfera Janos

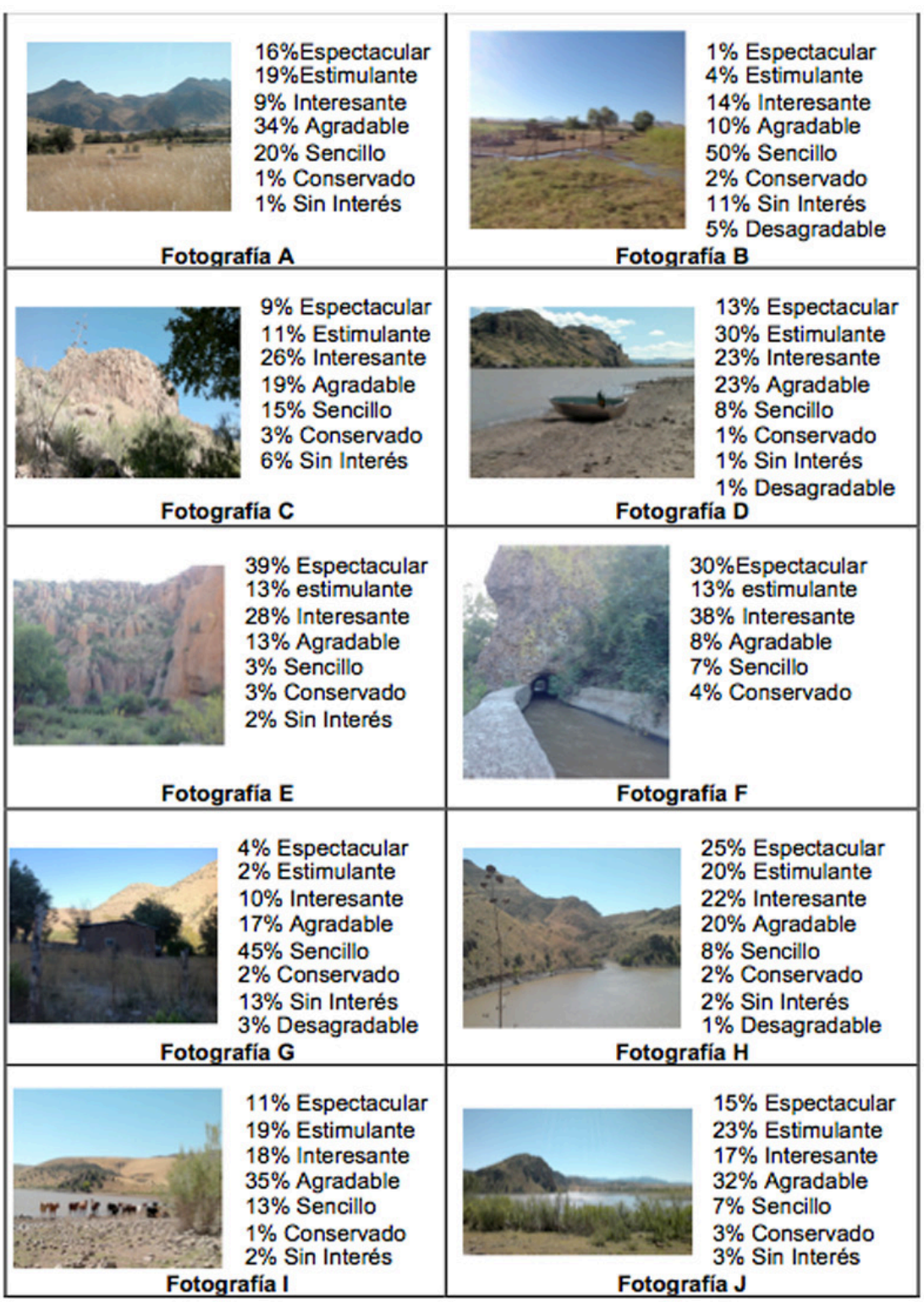

Fuente: Elaboración propia. 


\section{Comentario} FINAL

E s importante señalar que los resultados que se exponen son parciales, ya que actualmente se trabaja en el análisis de la información para la presentación final del estudio.

No obstante, se puede constatar que las incidencias anteriormente expuestas justifican la ventaja de utilizar un estudio interpretativo-cualitativo para la evaluación del paisaje por medio de fotografías de los paisajes más representativos de la Reserva de la Biosfera de Janos y recuperar, a través de los cuestionarios, la opinión y valoración por parte visitantes, residentes y personas interesadas en conocer el área natural. Se complementa esta evaluación con entrevistas a residentes, funcionarios y prestadores de servicios, puesto que ellos modifican, transforman o preservan el paisaje (Bertocelo, 2011 en Souto, 2011), integrándole un valor social y aportando criterios para su valoración, convirtiendo al paisaje en recurso para la consolidación de la oferta turística en espacios naturales (dos Santos, 2011).

\section{Agradecimientos}

S e agradece a Lourdes y Guillermo Acosta, familia del Ejido Casa de Janos, quienes con su sencillez, hospitalidad y particular interés por el turismo en su comunidad brindaron todo el apoyo para la realización del trabajo de campo. La oportunidad de poder convivir y participar en la dinámica de la familia y la comunidad enriqueció aún más la observación y reconocimiento del área de estudio.

\section{REFERENCIAS BIBLIOGRÁFICAS}

ConAnp. (2000). Estrategia Nacional para un desarrollo sustentable del turismo y la recreación en las áreas protegidas de México. SEMARNAT/CONANP.

Conanp. (2013). Programa de Manejo Reserva de la Biosfera Janos (1a. ed., p. 176). México, D.F: SEMARNAT/CONANP.

Cuevas, T. (2006). Gestión del Turismo Alternativo: caso noroeste de Chihuahua México. www.esade.edu. Recueprado en febrero 28, 2013.

Del Risco, Y., \& Salinas, E. (2003). El cobro de impuestos en las áreas protegidas como vía para el desarrollo sustentable. In A. Capacci (Ed.), Paisaje, ordenamiento territorial y turismo sostenible (pp. 55-68). Genova: Bringati.

Dos Santos, P. (2011). Marco teórico-metodológico de los estudios del paisaje: Perspectivas de aplicación en la planificación del turismo. Estudios Y Perspectivas En Turismo, 20(3), 522-541.

Dudley, N. (editor). (2008). Directrices para la aplicación de las categorías de gestión de áreas protegidas. (UICN, Ed.) (p. 96). Gland, Suiza.

Ferrer, L. (2010). Joan Nogué i Font: "Sin paisaje no hay turismo." Diario de Ibiza. Ibiza.

Gros, C. (2002). La relación "Paisaje-turismo-desarrollo local”: Examen de su significado en publicaciones recientes de divulgación territorial. Revista de Desarrollo Rural y Cooperativismo Agrario, 6, 123-133

Guerrero, E. (2011). México. El paraíso de los pinos, robles y cactus. In J.
Elbers (Ed.), Las áreas protegidas de América Latina América Latina: Situación actual y perspectivas para el futuro (pp. 69-78). Quito, Ecuador: UICN.

Iglesias, C. (Coord. . (2010). Estudios de Paisaje: Ámbitos dde Estudio y aplicaciones prácticas. (ECOPÁS, Ed.) (p. 145). Madrid, España: ECOPÁS.

INEGI. (2011). Instituto Nacional de estadística y geografía. México en Cifras. Recuperado abril 16, 2014, de http://www.inegi.org.mx/

López, N. (Coord. . (2012). Comunidades y Áreas silvestres protegidas: identidad, convivencias y conservación ambiental. (C. R. Heredia \& UNA-IDESPO, Eds.) (II., p. 235).

Maderuelo, J. (2005). El Paisaje : génesis de un concepto. Madrid :: Abada.

Millán, M. (2001). Interrelación entre la actividad turística y los espacios naturales protegidos. Redalyc Cuadernos de Turismo, 7, 93-110.

Municipio de Janos Chih. (2010). Plan Municipal de Desarrollo 20102013 Municipio de Janos Chihuahua. Plan Municipal de Desarrollo. Recuperado abril16, 2014, de http://www.janos.gob.mx/ atach2/janos/uploads/File/Janos Plan de Desarrollo Municipal.pdf

Muñoz, J. C. (2008). El turismo en los espacios naturales protegidos, algo más que una moda reciente. Boletin de la Asociación de Geográfos Españoles, 46, 291-304.

Muñoz-Pedreros, A. (2004). La evaluación del paisaje: una herramienta de gestión ambiental Landscape evaluation: an environmental management. Revista Chilena de Historia Natural, 77, 139-156. 
Nogué, J. (1989). Paisaje y turismo. (C) Estudios Turísticos, 1989, núm.103, p.35-45.

Pacheco, J., Ceballos, G., \& List, R. (2000). Los mamíferos de la Región de Janos-Casas Grandes, Chihuahua, México. Revista Mexicana de Mastozoología, 4, 69-83.

Pintó, J. (2005). Paisatge i activitat Turística. Atles del Turisme a Catalunya. Recuperado enero 18, 2013, de http://www.atlesturismecatalunya. cat/

Souto, P. (2011). Paisajes en la geografía contemporánea: concepciones y potencialidades. Revista Geográfica de América Central, (Número Especial EGAL 2011), 1-23.

UICN. (2009). IUCN-¿Qué es un área protegida? UICN. Recuperado abril 08, 2014, de http://www.iucn. org/es/sobre/union/secretaria/ oficinas/sudamerica/sur_trabajo/ sur_aprotegidas/ap_quees.cfm 